(R,K)-X-S or (R,K)-(R,K)-X-X-S for adenosine 3', 5'-monophosphate (CAMP)-dependent phosphorylation sites [B. E. Kemp, D. J. Graves, E. Benjamini, E. G. Krebs, J. Biol. Chem. 252, 4888 (1977)] and (S,T)-X-(R,K) for protein kinase C (PKC)-dependent phosphorylation [J. R. Woodget, K. L. Gould, T. Hunter, Eur. J. Biachem. 161, 177 (1986); A. Kishimcow a al, $J$. Bial. Chem 260, 12492 (1985)].

36. M. Noda et al., Nacure 320, 188 (1986).

37. We dedicate this paper to the memory of Tom Elkins, our colleague and friend whose pioncering studies provided the groundwort for this analysis. We thank C. Petersen, and R. Kreber for technical assistance; F. Blattuer, L. Marr, and members of the Blattuer lab for help with sequencing; E. Knust and
J. Campos-Ortega for providing stocts and clones; J. Tamkun, T. Schwar, L. Jan, Y. N. Jan, B. Hamition, and E. Meyerowitz for providing libraries; and our colleagues for advice and comments on the manuscripe. Supported by NIH grant NS15390, the Martey Charinble Trust, and a Klingenstein Fellowship to B.G N.S.A received support from NIH grant T32 GM07131 and the Muscular Dystrophy Association. G.A.R roceived support from the American Hear Association of Wisconsin, NIH grant T32 GM07131. and an NIH postdoctoral fellowship. This is paper number 3194 from the Laboratory of Genctios, University of Wisconsin-Madison.

3 May 1991; accopted 18 June 1991

\title{
Progressive Encephalopathy and Myopathy in Transgenic Mice Expressing Human Foamy Virus Genes
}

Katrin Bothe, Adruano Aguzzi, Hans lassmann, AXEL RETHWILM, IVAN HORAK*

Transgenic mice carrying the bel region of human foamy retrovirus (HFV) under transcriptional control of its own long terminal repeat expressed the transgene in their central nervous systems and in smooth and striated muscle tissues. The animals developed a progressive degenerative disease of the central nervous system and of the striated muscle. Because expression of the transgene was closely correlated with the appearance of structural damage and inflammatory reactions were scanty, the disease is likely to be caused directly by the HFV proteins. These unexpected findings call for a reevaluation of the pathogenic potential of HFV in humans.

$\mathrm{H}$ FV IS A RETROVIRUS ORIGINALLY isolated 20 years ago from individ: uals with various diseases $(1,2)$. Although the prevalence of HFV was reported to be high in certain geographical areas (3), it has not been possible to identify HFV as the causative agent of any human disease (4). As with the human T cell lymphoma virus (HTLV) and human immunodeficiency virus (HIV), the HFV genome has, in addition to structural retroviral genes, a region containing three open reading frames, bel-1 to bel-3. Bel-1 has some homology to HIV-2 Tat protein and, similarty to HIV Tat and HTLV-I Tax, functions as a transcriptional transactivator $(5-8)$.

Transgenic mice (9) have proved useful in the dissection of the pathogenic potential of the regulatory genes of the human retroviruses HTLV-I and HIV (10). To analyze the biological activity of HFV regulatory proteins we generated transgenic mice with two constructs: (i) $\mathrm{pHFV}_{\text {Af }}$, which contains the complete HFV genome in a noninfectious

K. Bothe, A. Rethwilm, I. Horak, Institute of Virology and Immunobiology University of Würzbung, Versbacherstresse 7, 8700 Würzburg, Federal Republic of Germany.

A. Agrumi, Reseanch Institute of Molecular Pathology, A. Agumzi, Reseanch Institute of Molecular

Dr. Bohr Gasse 7, 1030 Vienna, Austria. strasse 17, 1090 Vienna, Austria.

"To whom correspondence should be addressed. form because of a frameshift mutation in the integrase domain that created a premature stop codon in pol, and (ii) $\mathrm{p} \Delta \mathrm{gpe}$, in which $2.3 \mathrm{~kb}$ of gag-pol and $1.6 \mathrm{~kb}$ of env were deleted (7) (Fig. 1A). In both constructs, expression is controlled by the HFV long terminal repeat (LTR). Eight and nine founder animals (11) were derived with $\mathrm{pHFV}_{\mathrm{Af}}$ and $\mathrm{p} \Delta \mathrm{gpe}$, respectively. Five $\mathrm{pHFV}_{\mathrm{Af}}$ and six p $\Delta$ gpe mice developed a neurological syndrome consisting of ataxia, spastic tetraparesis; and blindness. The symptoms in the $\mathrm{pHFV}_{\mathrm{As}}$ mice appeared as earty as 5 to 6 weeks after birth, progressed rapidly, and led to death within 4 to 6 woeks. The pAgpe transgenic mice displayed a later onset of the disease ( 1 to constructs used for microinjection. Both constructs have been shown to transactivate HFV transcription in cultured cells (8). Af, Af II; E, Eoo RI; H, Hind III; Hp, Hpa II; N, Noo I; Pv, Pvu II. (B) Expression of HFV-RNA in various tissues of transpenic mice. Mice 75, 253, 267 and 881 harbor the pAgpe transgene, and mouse 362 is transgenic for $\mathrm{PHFV}$, Lanes 1 to 9 were from mouse 75: lane 1, brain; lane 2, liver; lane 3 , lung; lane 4, heart; lane 5, thymus; lane 6 , kidney; lane 7, muscle; lane 8, lymph nodes; lane 9, spleen. Lane 10, mouse 362 brain; lane 11, mouse 253 testis; lane 12, mouse 253 brain; lane 13 , mouse 267 brain; lane 14, nontransgenic littermate brain; lane 15, nontransgenic littermate brain; lane 16, mouse 881 forebrain; lane 17 , mouse 881 parietal and midbrain; lane 18 , mouse 881 cerebellum and brain stem.
4 months of age) with a slower progression, which allowed their breeding. The remaining six founder animals, which were asymptomatic, did not express the transgene (12). Northern (RNA) hybridization analysis of the expression of the transgene revealod RNAs of sizes corresponding to a total transcript and to bel subgenomic mRNA only in the forebrain and in the cerebellum of all animals with disease symptoms (Fig. 1B)

In all animals expressing either construct, the pathological findings were restricted to the central nervous system (CNS) and to the striated muscle. The disease phenotype was transmitted to the offspring with a penetrance of $100 \%$. Animals with the clinical symptoms described above showed variable degrees of selective nerve cell degeneration in the forebrain, with tissue atrophy and reactive gliosis. The areas affected most frequently were the CA3 layer of the hippocampus and the telencephalic cortex (Fig. 2). Some lesions exhibited a more abnormal pattern, with incomplete cortical and subcortical necrosis and occasionally with macrophages and mild lymphocytic infiltrates in the meninges and in the perivascular spaces (13). In situ hybridization analysis revealed a complex pattern of expression in the CNS, with groups of neurons, oligodendrocytes, and astrocytes containing high amounts of transcript in the brain and spinal cord. The distribution of positive cells was bilaterally symmetrical. In animals 4 weeks old, most neurons in the CA3 sector of the hippocampus and a subpopulation of cortical neurons were strongly positive for HFV RNA, even in animals in which onset of symptoms had not yet occurred (Fig. 2A). In sick animals older than 6 weeks, most CA3 neurons had degenerated (Fig. 2B), and the highest levels of expression were seen in cortical neurons.

Two $\mathrm{pHFV}_{\mathrm{Af}}$ and four $\mathrm{p} \Delta \mathrm{gpe}$ mice showed focal degeneration of striated mus-

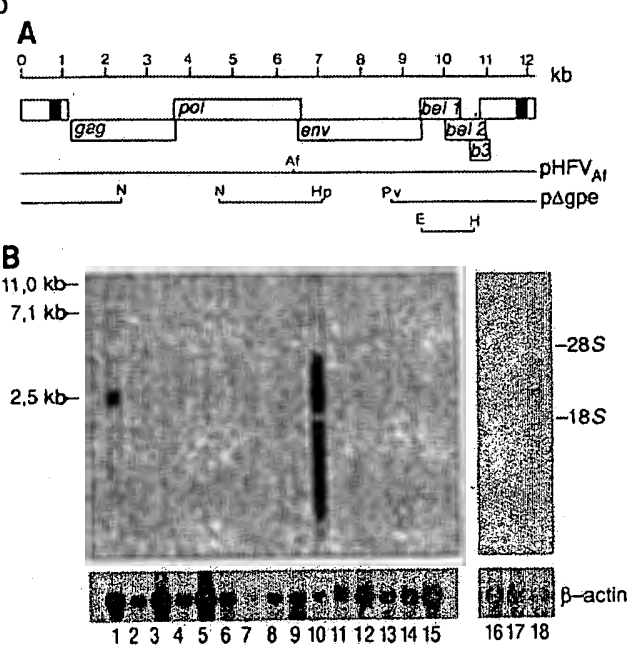


cles. These changes ranged from degeneration of single myotubes to large areas of myogenic atrophy with extensive necroses (Fig. 2D). In situ hybridization of cross sections of the affected striated muscles demonstrated that expression preceded degeneration and was confined to the myotubes.

Of the organs that did not show pathological changes, transgene expression could be detected by in situ hybridization in a small fraction of retinal interneurons and in a few smooth muscle cells in almost all organs analyzed (13). Higher numbers of positive cells were detected in the smooth muscle layer of the digestive tract.

In addition to the lesions described above, the $\mathrm{pHFV}_{\text {Af }}$ transgenic mice showed spotty symmetric areas of grayish color in the white matter because of severe damage of the myelinated tracts in the anterior commissure, the corpus callosum, the optic nerves, the brain stem, the cerebellar white matter, and, to a lesser extent, in the spinal cord. These lesions consisted of fluid-filled vacuoles in the white matter (Fig. 3). Immunocytochemistry for glial fibrillary acidic protein (GFAP) revealed a striking reactive gliosis in the affected areas, whereas oligodendrocytes showed only occasional swellings. Transgene-expressing cells were invariably clustered in the immediate proximity of the lesions. Some of these cells had the typical size and structure of oligodendrocytes, but a small fraction was identified as being composed of astrocytes by combined in situ hybridization for HFV RNA and immunohistochemistry for GFAP (13). The extent and time of onset of these lesions varied only slightly between individual animals and between families derived from distinct founders.

Fig. 2. Neuronal and muscle pathology in HFV transgenic mice (18). (A) In situ hybridization with an HFV probe of the hippocampus of a 4-week-old animal before onset of symptoms. Most neurons in the CA3 layer (arrowheads) expressed high levels of HFV RNA, whereas the $\mathrm{CAl}$ area (open arrows) and the dentate gyrus (Dg) were essentially negative (hematoxylin-eosin, 100X). (B) Selective nerve cell loss in the CA3 layer of the hippocampus in a 6-week-old animal. The lesioned segment is sharply demarcated and corresponds precisely to the area of HFV expression in the younger animal (Luxcol fast blue-Nissl stain, 100X). (C) Frontal cortex from a 7-weck-old transgenic animal (GFAP immunohistochemistry, 200X) showing loss of neurons. Several GFAP-positive reactive astrocytes (arrowheads) and activated microglial cells (arrows) are present. (D) Paravertebral striated muscles of a transgenic animal. There is severe myogenic atrophy with size variation of the muscle fbers, increased number of internal nuclei, loss of cross-striation, hyaline degeneration of the sarooplasm, and hypertrophy of adjacent myotubes. A majority of viable muscle fibers express HFV RNA
The observation that only a small fraction of cells in specific lineages expressed high levels of transgene RNA, whereas other cells of the same lineages were negative, is unusual for transgenes controlled by promoters with restricted tissue specificity, such as ret-
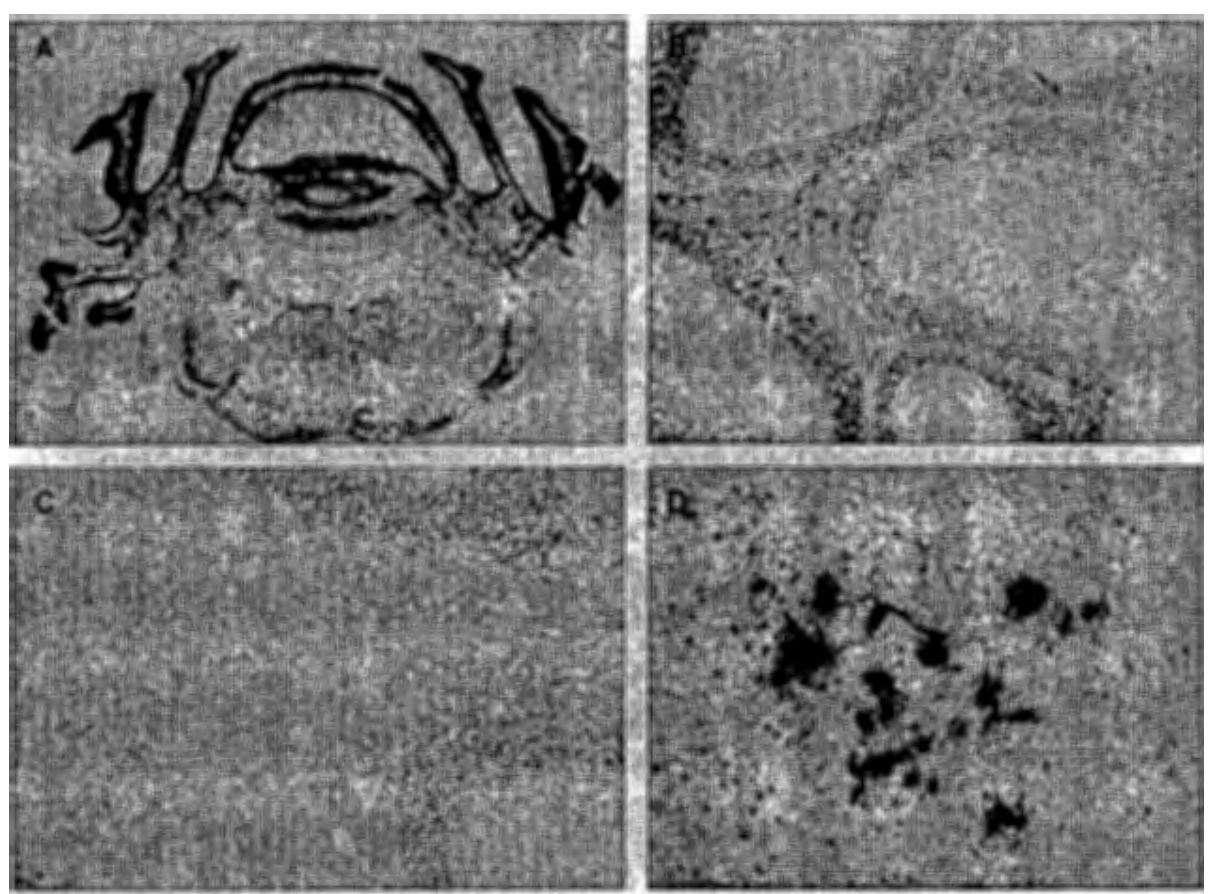

Fig. 3. White matter lesions. (A) Cross section through the cerebellum of a 97-day-old PHFV $_{A}$ transgenic animal. There is widespread vacuolar degeneration in the white matter of the cerebellum (Luxol fast blue-Nissl, 12,5X). (B) HFV expression in the cerebellum of the same animal (in situ hybridization, 50x). Most cells expressing HFV RNA are located in the white matter and are closely associated with the microcystic changes. Some positive cells are present in the granular layer. (C) Immunohistochemical demonstration of myelin basic protein (MBP) (100x). In the immediate vicinit of the vacuoles, MBP immunoreactivity is essentially preserved and virtually no macrophages witt myelin degradation products are present, indicating that structural loss and degradation of myelin ha: not yet taken place. (D) HFV expression in the anterior commissure. Large amounts of transgenic RNA are present in the cells of the anterior commissure but not in the neurons of the surrounding basa ganglia (in situ hybridization, 200x).
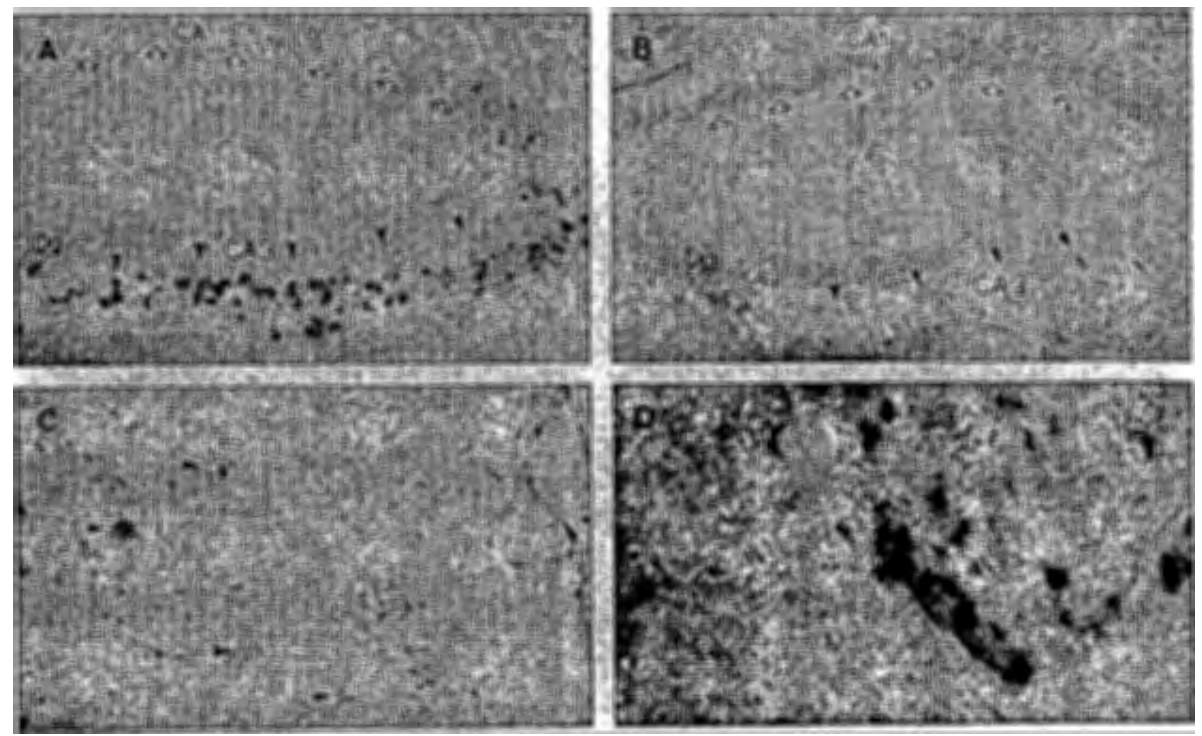

SCIENCE, VOL. 25:

roviral LTRs. Mosaicism and positional ef-
fects of the transgene integration could br excluded because these observations wer
confirmed in founder animals from 11 inde pendent integration events and in their off spring. Also, we found no evidence fol

$\sqrt{2}$


reversion of the constructs to an infectious form or for horizontal spread of HFV (13). It is unlikely that expression of the HFV constructs depends on the cell cycle because neurons are irreversibly postmitotic in adult animals. These observations indicate that additional, unknown cellular factors may be required for high levels of HFV LTR-directed transcription.

Because expression of HFV was predominantly seen in anatomical structures that will eventually degenerate, it is likely that the gene products of $\mathrm{HFV}$ are directly responsible for the observed lesions. However, we are unable to identify conclusively which proteins of HFV are responsible for neurotoxicity. Nerve cell degeneration was present in animals carrying the pAgpe DNA, which essentially contains only the bel reading frames. A possible candidate for neurotoxicity is the bel-1 gene product, which is functionally comparable to the retroviral transactivating factors, HIV tat and HTLV-I tax (5-7). However, transgenic mice carrying LTR tat and LTR tax develop Kaposi sarcomas and peripheral neurogenic and mesenchymal tumors, respectively (10), but no degenerative encephalopathy. These differences may relate to the tissue-specific expression pattern caused by the HFV cis regulatory elements or to different cellular and molecular targets of the bel-1 protein.

The pathologies observed in HFV mice are reminiscent of human retroviral disease of the CNS: neuronal loss and white matter damage are often present in HIV-associated encephalopathy (14) and vacuolar myelopathy (15) and in HTLV-I-induced disease of the CNS (16). The mechanisms of neurotoxicity operating in these conditions may be related to those leading to neurological damage in HFV transgenic mice. In fact, one of the original isolates of HFV was obtained from the brain of a patient with a neurodegenerative disease with features reminiscent of the lesions in the HFV mice (2).

Athough a specific pathology has not been unequivocally associated with HFV infection, and foamy viruses generally have been regarded as benign (4), detection of HFV in human diseases might be problematic if virulence is restricted to immunocompromised hosts or to abortive forms of infection (17). The findings described here prompt a critical reappraisal of the potential role of HFV as a human pathogen. It will be crucial to search for evidence of HFV infection, or superinfection, in neurodegenerative and myopathic disorders of unknown etiology and neurological syndromes in immunodeficient patients.

\section{REFERENCES AND NOTES}

1. G. Achong et al., J. Natl. Cancer Inst. 42, 299 (1971); D. Young et al., Arch. Gesamte Vinusforsch. 42, 228 (1973)

2. K. R. Cameron et al., Lancet ii, 796 (1978).
3. R. H. Muller et al., J. Gen. Virol. 47, 399 (1980). 4. R. A. Weiss, Nature 333, 497 (1988); B. N. Fields and D. M. Knipe, in Virology, B. N. Fields et al. Eds. (Raven, New York, 19\%0)

5. R. M. Flügel et al., EMBO J. 6, 2077 (1988).

6. A. Rethwilm et al., Virology 175, 568 (1990).

7. A. Rethwilm et al., Proc. Natl. Acad. Sci. U.S.A. 88 941 (1991)

8. A. Rethwilm et al., Nucleic Acids Res. 18, 733 (1990)

9. R. Jaenisch Science 240, 1468 (1988); D. Hanahan ibid. 246, 1265 (1989).

10. J. Vogel et al., Nature 335, 606 (1988); M. Nerenberg, S. H. Hinrichs, R. K. Reynolds, G. Khoury, G. Jay, Science 237, 1324 (1987); S. H. Hinrichs, M. Nerenberg, R. K. Reynolds, G. Khoury, G. Jay, ibid., p. 1340.

11. Transgenic mice were derived by pronuclear microinjection of (C57BL/6 $\times \mathrm{C} 3 \mathrm{H}) \mathrm{F}_{2}$ fertilized eggs as described [B. Hogan et al., in Manipulating the Mouse Embryo: A Laboratory Manual, B. Hogan et al., Eds. (Cold Spring Harbor Laboratory Press, Cold Spring Harbor, NY, 1986)]. Eight PHFV and nine $\mathrm{p} \Delta \mathrm{gpe}$ transgenic animals were obtained from 87 and 93 newborns, respectively. The number of integrated copies of the constructs varied from 3 to 30 per haploid genome (12). Total RNA (15 $\mu \mathrm{g}$ per lane) was separated on $1.5 \%$ formaldehydeagarose gels, transferred to a nylon membrane, and hybridized to an Eco RI-Hind III fragment encoding nucleotides 9475 to 10697 of HFV (Fig. 1A). Blots were rehybridized with a $\beta$-actin probe for quantification of the amount of RNA

12. K. Bothe et al., unpublished data.
13. A. Aguzzi et al., unpublished data.

14. F. Giangaspero et al., Arta Neumpachol. 78, 662 (1989); S. Ketzler et al., ibid. 80, 92 (1990); P. Kleihues et al., ibid. 68, 333 (1985).

15. C. K. Petito et al., N. Engl. J. Med. 312, 874 (1985)

16. P. Piccardoet al., Ann. Neurol. 23 (suppl.), 156 (1988).

17. A. H. Sharpe et al., Nature 346, 181 (1990)

18. Twenty HFV transgenic mice from 11 independent families (founders, FI and F2 animais) were analyzed histologically. Animals were killed by cardiac perfusion with $4 \%$ buffered formaldehyde. Organs were fixed, briefly decalcified with acid and embedded in paraffin by standard procedures. Sections (4 $\mu \mathrm{m}$ thick) were stained with hematoxylin-eosin. Peroxidase immunohistochemistry was performed as described [A. Aguzzi et al., Oncogene 6, 113 (1991)]. Rabbit antisera to myelin basic protein and to GFAP (DAKO, Copenhagen) were used. In situ hybridizations were performed according to A Aguzzi and co-workers [New Biol. 2, 533 (1990)]. ${ }_{35} \mathrm{~S}$-labeled RNA probes were generated with the Eco RI-Hind III fragment of HFV described above. Sense-transcribed RNA probes and an osteonectin probe were used as controls [P. W. Hol. land et al., J. Cell Biol. 105, 473 (1987)]

19. We thank E. F. Wagner, O. D. Wiestler, and S. Siddell for support, discussions, and critical reading of the manuscript, and H. Haber and K. Gruber for their invaluable assistance. Supported by the Deutsche Forschungsgemeinschaft (SFB 165) and by Wilhelm Sander-Stiftung.

22 February 1991; accepted 20 May 1991

\section{Identification of Profilin as a Novel Pollen Allergen; IgE Autoreactivity in Sensitized Individuals}

\section{Rudolf Valenta, Michael Duchéne, Karin Pettenburger, Christian Sillaber, Peter Valent, Peter Bettelheim, Michael Breitenbach, Helmut Rumpold, Dietrich Kraft, OTTO SCHEINER*}

A complementary DNA encoding a pollen allergen from white birch (Betula verrucosa) that was isolated from a pollen complementary DNA library with serum immunoglobulin E from a birch pollen-allergic individual revealed significant sequence homology to profilins. The recombinant protein showed high affinity to poly-L-proline. Immunoglobulin $\mathbf{E}$ antibodies from allergic individuals bound to natural and recombinant birch profilin and also to human profilin. In addition, birch and human profilin induced histamine release from blood basophils of profilin-allergic individuals, but not of individuals sensitized to other plant allergens. The structural similarity of conserved proteins might therefore be responsible for maintaining immunoglobulin $\mathbf{E}$ antibody titers in type I allergy.

A LLERGIES OF THE IMMEDIATE TYPE are a major health problem in industrialized countries, where up to

R. Valenta, K. Pettenburger, D. Kraft, O. Scheiner, Institute of General and Experimental Pathology, University of Vienna, A-1090 Vienna, Austria.

versity of Vienna, A-1090 Vienna, Austria. M. Duchene, Institute of General and Experimental Tropical Medicine, University of Vienna, A-1095 Vi. enna, Austria.

C. Sillaber, P. Valent, P. Bettelheim, First Medica Department, University of Vienna, A-1090 Vienna, Aus. tria.

M. Breitenbach, Institute of Microbiology and Genetics, University of Vienna, A-1090 Vienna, Austria.

$H$. Rumpold, Institute of Clinical Chemistry and Labo H. Rumpold, Institute of Clinical Chemistry and Labo-
ratory Diagnostics, University of Vienna, A-1090 Vienna, Austria.

To whom correspondence should be addressed.
$15 \%$ of the population suffers from type I allergic symptoms (rhinitis, conjunctivitis, and bronchial asthma). An understanding of the complex pathogenetic mechanisms leading to allergy requires information about the structure and function of allergenic proteins. Comparison of the sequences of cloned allergens with those of known proteins has already contributed to the characterization of some allergenic proteins. The major birch (Betula verrucosa) pollen allergen Bet v I (1) and the white-faced hornet venom allergen antigen $5 \mathrm{Dol} \mathrm{m} \mathrm{V} \mathrm{(2)} \mathrm{were} \mathrm{shown} \mathrm{to} \mathrm{be}$ highly homologous to pathogenesis-related plant proteins. The major house dust mite allergen Der $p I$ is homologous to proteases- 